Few-Body Systems 0, 1-4 (2019)

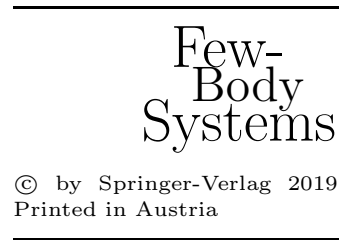

\title{
Color Gauge Invariance in Hard Processes *
}

\author{
F. Pijlman \\ Vrije Universiteit, Department of Physics, Amsterdam
}

\begin{abstract}
Within the theoretical framework we apply, a suggested origin for single spin asymmetries is the presence of gauge links in transverse momentum dependent distribution functions. Recently we found new gauge link structures in a number of hard processes. These structures need to be considered in the evolution of parton distribution functions and for establishing factorization.
\end{abstract}

\section{Soft matrix elements in semi-inclusive DIS and Drell-Yan}

The description of the internal structure of the nucleon is fairly well understood in terms of transverse momentum integrated distribution functions in contrast to the unintegrated ones. Within the formalism the latter provide access to the transverse spin of quarks and moreover, they are sensitive to the average gluon field in the nucleon which can be measured in single spin asymmetries (SSA's). Following the approach and notations of Ref. [1], we will try to understand how these distribution functions are defined in the parton model, where it is assumed that partons in the nucleon are soft.

In semi-inclusive DIS (SIDIS), see Fig 1 the virtual photon couples to the current sandwiched between the in- and outgoing hadrons. The cross-section is proportional to the leptonic tensor, $L_{\mu \nu}$, contracted with the hadronic tensor, $W^{\mu \nu} \sim{ }_{\text {in }}\left\langle P\left|J^{\mu}(0)\right| P_{X}, P_{h}\right\rangle_{\text {out out }}\left\langle P_{X}, P_{h}\left|J^{\nu}(0)\right| P\right\rangle_{\text {in }}$. Assuming factorization one can show that in the parton model the virtual photon actually scatters off a quark for which one of the leading contributions in $M / Q$ is given in Fig 1 Other contributions at this order involve longitudinally polarized gluons and gluon fields at infinity. Both contributions are needed for a color gauge invariant description, but we will discard them for the moment. The result for the hadronic tensor, expressed in Fourier transformed non-perturbative matrix elements, is

$$
\begin{aligned}
W^{\mu \nu} & =\int \mathrm{d}^{4} p \mathrm{~d}^{4} k \delta^{4}(p+q-k) \operatorname{Tr}\left[\Phi(p) \gamma^{\mu} \Delta(k) \gamma^{\nu}\right], \\
\Phi_{i j}(p) & =\mathcal{F} \mathcal{T} \operatorname{in}\left\langle P, S\left|\bar{\psi}_{j}(0) \psi_{i}(\xi)\right| P, S\right\rangle_{\text {in }}, \\
\Delta_{i j}(k) & =\sum_{X} \mathcal{F} \mathcal{T}\left\langle 0\left|\psi_{i}(\xi)\right| P_{h}, S_{h} ; X\right\rangle_{\text {out out }}\left\langle P_{h}, S_{h} ; X\left|\bar{\psi}_{j}(0)\right| 0\right\rangle .
\end{aligned}
$$

*Presented at Light-Cone 2004, Amsterdam, 16 - 20 August 


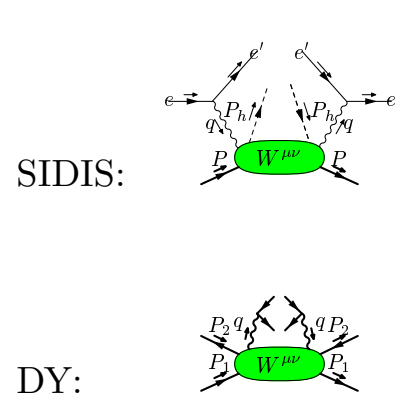

a

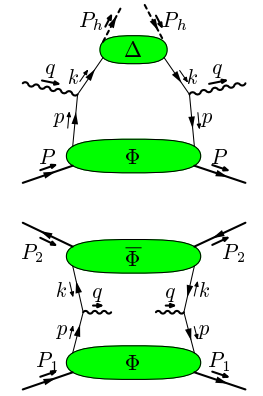

$\mathrm{b}$

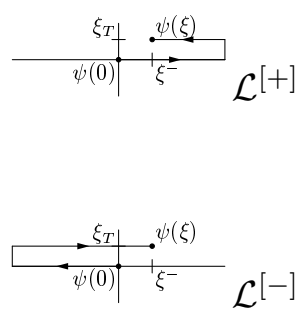

c

Figure 1. a) cross-section, b) one of the leading contributions in the parton model, c) gauge links.

When considering the Drell-Yan process (DY), see Fig 1 the hadronic tensor in the parton model reads

$$
\begin{aligned}
W^{\mu \nu} & =\int \mathrm{d}^{4} p \mathrm{~d}^{4} k \delta^{4}(p+k-q) \operatorname{Tr}\left[\Phi(p) \gamma^{\mu} \bar{\Phi}(k) \gamma^{\nu}\right], \\
\Phi_{i j}(p) & =\mathcal{F} \mathcal{T}_{\text {in }}\left\langle P, S\left|\bar{\psi}_{j}(0) \psi_{i}(\xi)\right| P, S\right\rangle_{\mathrm{in}} .
\end{aligned}
$$

Without the discarded gluon contributions we find that the non-perturbative matrix elements are process independent. To prove the universality of these matrix elements, one should take gluon contributions into account and show that the processes factorize into a hard and a universal soft part (the non-perturbative information). In such proofs infrared divergences, appearing at higher order in the coupling constant, have to be absorbed in the soft parts in a universal manner [2]. Recently this topic received a lot of attention [3, 4].

\section{Single spin asymmetries and gauge links}

Recent measurements [5] with polarized electrons and unpolarized hadrons show significant SSA's. In this case the asymmetry is proportional to the antisymmetric part of the leptonic tensor contracted with the hadronic tensor. The derivation

$$
\begin{aligned}
& W^{\mu \nu} \sim \operatorname{in}\left\langle P\left|J^{\mu}(0)\right| P_{X}, P_{h}\right\rangle_{\text {out }} \text { out }\left\langle P_{X}, P_{h}\left|J^{\nu}(0)\right| P\right\rangle_{\text {in }} \\
& ={ }_{\text {out }}\left\langle P_{X}, P_{h}\left|J^{\mu \dagger}(0)\right| P\right\rangle_{\text {in }}^{*} \text { in }\left\langle P\left|J^{\nu \dagger}(0)\right| P_{X}, P_{h}\right\rangle_{\text {out }}^{*} \\
& =\operatorname{in}\left\langle P\left|\mathcal{T}^{\dagger} \mathcal{T} J^{\nu \dagger}(0) \mathcal{T}^{\dagger} \mathcal{T}\right| P_{X}, P_{h}\right\rangle_{\text {out }}^{*} \quad \text { out }\left\langle P_{X}, P_{h}\left|\mathcal{T}^{\dagger} \mathcal{T} J^{\mu \dagger}(0) \mathcal{T}^{\dagger} \mathcal{T}\right| P\right\rangle_{\text {in }}^{*} \\
& \neq^{\mathcal{T}} \quad \text { in }\left\langle\bar{P}\left|\mathcal{T} J^{\nu \dagger}(0) \mathcal{T}^{\dagger}\right| \bar{P}_{X}, \bar{P}_{h}\right\rangle_{\text {out }} \quad \text { out }\left\langle\bar{P}_{X}, \bar{P}_{h}\left|\mathcal{T} J^{\mu \dagger}(0) \mathcal{T}^{\dagger}\right| \bar{P}\right\rangle_{\text {in }} \\
& ={ }^{\mathcal{P}} \quad{ }_{\text {in }}\left\langle P\left|\mathcal{P}^{\dagger} \mathcal{T}^{\dagger} J^{\nu \dagger}(0) \mathcal{T} \mathcal{P}\right| P_{X}, P_{h}\right\rangle_{\text {out out }}\left\langle P_{X}, P_{h}\left|\mathcal{P}^{\dagger} \mathcal{T}^{\dagger} J^{\mu \dagger}(0) \mathcal{T} \mathcal{P}\right| P\right\rangle_{\text {in }} \\
& \sim W^{\nu \mu} \text {, }
\end{aligned}
$$

shows that the hadronic tensor can have an antisymmetric part because under time reversal out-states transform into spatial momentum reversed (e.g. $P \rightarrow \bar{P}$ ) in-states $\mathcal{T}\left|P_{X}, P_{h}\right\rangle_{\text {out }}=\left|\bar{P}_{X}, \bar{P}_{h}\right\rangle_{\text {in }} \neq\left|\bar{P}_{X}, \bar{P}_{h}\right\rangle_{\text {out }}$. The possible mechanism of final state interactions in quark fragmentation was suggested as an explanation in the parton model $[\underline{6}$. 


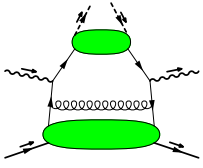

$\mathrm{a}: \mathcal{L}^{[+]}$

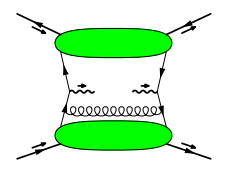

b: $\frac{3}{8} \mathcal{L}^{[+]} \operatorname{Tr} \mathcal{L}^{[\square]}-\frac{1}{8} \mathcal{L}^{[-]}$

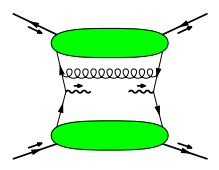

c: same as b

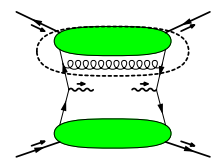

d: ?

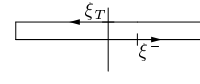

e: $\mathcal{L}^{[\square]}$

Figure 2. The gauge links for lower blobs in several diagrams

A decade later, a model calculation showed that SSA's can be generated without considering the fragmentation of quarks [7. This was a sign for a new source for SSA's which was missing in the parton model description. Within the formalism outlined in section 1, this same effect can be attributed to gluon contributions. The longitudinally polarized gluons can be resummed, giving a gauge link running from each quark field to infinity for SIDIS [8]. The fact that in DY the gauge link runs via minus infinity was not considered to be important since in the light-cone gauge the links would vanish. The other leading contributions are the gluon fields at infinity and they turned out to be the missing part in the parton model description. It was shown that one can resum those contributions as well, giving a transverse link 9]. Taking all these effects into account one finds that the leading order expression for the hadronic tensors only differs by the presence of a gauge link $(\mathcal{L})$ in the matrix elements (see Fig $\mathbb{1}$ )

$$
\begin{aligned}
\text { SIDIS : } & \Phi_{i j}(p) \rightarrow \Phi_{i j}^{[+]}(p)=\mathcal{F} \mathcal{T}\left\langle P, S\left|\bar{\psi}_{j}(0) \mathcal{L}^{[+]}(0 ; \xi) \psi_{i}(\xi)\right| P, S\right\rangle, \\
\text { DY : } & \Phi_{i j}(p) \rightarrow \Phi_{i j}^{[-]}(p)=\mathcal{F} \mathcal{T}\left\langle P, S\left|\bar{\psi}_{j}(0) \mathcal{L}^{[-]}(0 ; \xi) \psi_{i}(\xi)\right| P, S\right\rangle .
\end{aligned}
$$

One can even obtain a color gauge invariant description at next-to-leading order in $M / Q$ [1, 10] from which explicit asymmetries in SIDIS were obtained [1].

Having different but explicit gauge invariant matrix elements leads to several interesting properties. For instance, assuming factorization in SIDIS and DY one can still relate their matrix elements via a time reversal operation. It is possible to divide the matrix elements in a process independent group (called T-even) and a process dependent group (called T-odd) where the latter changes sign when comparing SIDIS and DY at this order. The last group is proportional to the gluon field [1], $\mathcal{F} \mathcal{T}\left\langle P, S\left|\bar{\psi}_{j}(0)\left[\int_{-\infty}^{\infty} \mathrm{d} \eta^{-} \mathcal{L}\left(0, \eta^{-}\right) G^{+\alpha}\left(\eta^{-}\right) \mathcal{L}\left(\eta^{-}, \xi^{-}\right)\right] \psi_{i}(\xi)\right| P, S\right\rangle$, and can be a source for SSA's. For fragmentation matrix elements with different links it is complicated to derive such a relation due to the combination of final state interactions and gauge links. More information is given in Ref. 11, 4, 12.

\section{Recent developments}

Interesting effects occur when considering $\alpha_{s}$ corrections in SIDIS and DY. It was shown that closed gauge links, $\mathcal{L}^{[\square]}$, in distribution matrix elements of a considered nucleon can only appear if there are other incoming and outgoing QCD (bound) states [13. Therefore, such Wilson loops do not appear in the nucleon in SIDIS because in that case there are, besides the nucleon, only outgoing QCD states even when gluon bremsstrahlung is considered. As a result, the link 
still runs via plus infinity (see Fig 2 ). Supported by a first order calculation, the link very likely runs via plus infinity for the vertex correction and the self energy of the quark as well. However, gluon bremsstrahlung in DY, where there are incoming hadrons, creates outgoing QCD states. In that case the link turns out to depend on the number of gluons radiated (see Fig $2 \mathrm{~b}, \mathrm{c}$ ). For the vertex and self energy correction alone, the link probably still runs via minus infinity.

If the difference of gauge links matters (e.g. nonzero Sivers function), then the behavior of links under gluon radiation may imply that the evolution of distribution functions in DY is not the same as in SIDIS. This questions the universality of these functions. Moreover, to prove factorization one has to absorb the infrared divergence in Fig $2 \mathrm{~d}$ into the upper blob. Since the radiated gluon also affects the link of the lower blob, it will be difficult - if not impossible - to factorize such diagrams.

At this moment it is unclear whether transverse momentum dependent effects in DY can be factorized. In $e^{+} e^{-}$annihilation, where hadrons are only in the final state and therefore gauge links do not change under gluon radiation, such a factorization proof does exist [14. It is expected that the incorporation of gauge links will be an essential ingredient in considerations on factorization.

This work, done in collaboration with D. Boer, C. Bomhof and P. Mulders, was presented at a nice conference for which the organizers are acknowledged.

\section{References}

1. D. Boer, P.J. Mulders, F. Pijlman, Nucl.Phys.B 667, 201 (2003)

2. R.K. Ellis et al., Nucl.Phys.B 152, 285 (1979)

3. X. Ji, J. Ma and F. Yuan, hep-ph/0404183 and Phys.Lett.B 597, 299 (2004) A. Idilbi, X. Ji, J. Ma and F. Yuan, hep-ph/0406302

4. J.C. Collins and A. Metz, hep-ph/0408249

5. H. Avakian et al., Phys.Rev.D 69, 112004 (2004), E. Avetisyan et al., hep-ex/0408002

6. J.C. Collins, Nucl.Phys.B 396, 161 (1993)

7. S.J. Brodsky, D.S. Hwang and I. Schmidt, Phys.Lett.B 530, 99 (2002)

8. A.V. Efremov and A.V. Radyushkin, Theor. Math. Phys. 44, 774 (1981)

9. A.V. Belitsky, X. Ji and F. Yuan, Nucl.Phys.B 656, 165 (2003)

10. D. Boer, P.J. Mulders, Nucl.Phys.B 569, 505 (2000)

11. A. Bacchetta, P.J. Mulders and F. Pijlman, Phys.Lett.B 595, 309 (2004)

12. A. Metz, Phys.Lett.B 549, 139 (2002)

13. C.J. Bomhof, P.J. Mulders and F. Pijlman, Phys.Lett.B 596, 277 (2004)

14. J.C. Collins and D.E. Soper, Nucl.Phys.B 193, 381 (1981) 